\title{
Improved oscillation results for second-order half-linear delay differential equations
}

\author{
George E. Chatzarakis ${ }^{1}$, Irena Jadlovská*2 \\ ${ }^{1}$ Department of Electrical and Electronic Engineering Educators, School of Pedagogical and Technological \\ Education (ASPETE), 14121, N. Heraklio, Athens, Greece \\ ${ }^{2}$ Department of Mathematics and Theoretical Informatics, Faculty of Electrical Engineering and \\ Informatics, Technical University of Košice, Letná 9, 04200 Košice, Slovakia
}

\begin{abstract}
In this paper, we study the second-order half-linear delay differential equation of the form

$$
\left(r(t)\left(y^{\prime}(t)\right)^{\alpha}\right)^{\prime}+q(t) y^{\alpha}(\tau(t))=0 .
$$

We establish new oscillation criteria for $(E)$, which improve a number of related ones in the literature. Our approach essentially involves establishing sharper estimates for the positive solutions of $(E)$ than those presented in known works and a comparison principle with first-order delay differential inequalities. We illustrate the improvement over the known results by applying and comparing our method with the other known methods on the particular example of Euler-type equations.
\end{abstract}

Mathematics Subject Classification (2010). 34C10, 34K11

Keywords. half-linear differential equation, delay, second-order, oscillation

\section{Introduction}

Consider the second-order half-linear delay differential equation of the form

$$
\left(r(t)\left(y^{\prime}(t)\right)^{\alpha}\right)^{\prime}+q(t) y^{\alpha}(\tau(t))=0, \quad t \geq t_{0}>0 .
$$

Throughout the paper, it is assumed that the following conditions hold:

(i) $\alpha>0$ is a quotient of odd positive integers;

(ii) $\tau \in \mathcal{C}^{1}\left(\left[t_{0}, \infty\right)\right), \tau^{\prime}(t)>0, \tau(t) \leq t$ and $\lim _{t \rightarrow \infty} \tau(t)=\infty$;

(iii) $q \in \mathcal{C}\left(\left[t_{0}, \infty\right)\right)$ is nonnegative and does not vanish identically on any half line of the form $\left[t_{*}, \infty\right)$;

(iv) $r \in \mathfrak{C}^{1}\left(\left[t_{0}, \infty\right)\right)$ is positive and satisfies

$$
R\left(t, t_{0}\right):=\int_{t_{0}}^{t} r^{-1 / \alpha}(s) \mathrm{d} s \rightarrow \infty \quad \text { as } \quad t \rightarrow \infty
$$

\footnotetext{
*Corresponding Author.

Email addresses: geaxatz@otenet.gr (G. E. Chatzarakis), irena.jadlovska@tuke.sk (I. Jadlovská)

Received: 24.08.2017; Accepted: 17.10.2017
} 
Under the solution of equation $(E)$ we mean a function $y \in \mathcal{C}\left(\left[t_{a}, \infty\right), \mathbb{R}\right)$ with $t_{a}=\tau\left(t_{b}\right)$, for some $t_{b} \geq t_{0}$, which has the property $r\left(y^{\prime}\right)^{\alpha} \in \mathcal{C}^{1}\left(\left[t_{a}, \infty\right), \mathbb{R}\right)$ and satisfies $(E)$ on $\left[t_{b}, \infty\right)$. We consider only those solutions of $(E)$ which exist on some half-line $\left[t_{b}, \infty\right)$ and satisfy the condition $\sup \left\{|x(t)|: t_{c} \leq t<\infty\right\}>0$ for any $t_{c} \geq t_{b}$.

As is customary, a solution $y(t)$ of $(E)$ is said to be oscillatory if it is neither eventually positive nor eventually negative. Otherwise, it is said to be nonoscillatory. The equation itself is termed oscillatory if all its solutions oscillate.

The problem of establishing oscillation criteria for differential equations with deviating arguments has been a very active research area over the past decades and several references and reviews of known results can be found in the monographs by Agarwal et al. [1-4], Došlý and Řehák [6] and Győri and Ladas [11].

The oscillation problem for $(E)$ and its particular cases (or its generalizations on dynamic, neutral, nonlinear equations, etc.) has been studied extensively, see, e.g., $[5,7,8,12,15-18,20-26]$ and the references therein.

One of the basic techniques in oscillation theory is to acquire criteria by comparing the given differential equation with first-order delay differential equations or inequalities, whose oscillatory behavior is known in advance.

The first results in this direction for second-order delay equations were given by Koplatadze [15] in 1986 and Wei [22] in 1988, who proved that the equation

$$
y^{\prime \prime}(t)+q(t) y(\tau(t))=0
$$

is oscillatory if

$$
\liminf _{t \rightarrow \infty} \int_{\tau(t)}^{t} q(s) \tau(s) \mathrm{d} s>\frac{1}{\mathrm{e}} .
$$

In 2000, Koplatadze, Kvinikadze and Stavroulakis [14, Theorem 1] presented an improved oscillation criterion for (1.1), namely,

$$
\liminf _{t \rightarrow \infty} \int_{\tau(t)}^{t}\left(\tau(s)+\int_{t_{0}}^{\tau(s)} \xi \tau(\xi) q(\xi) \mathrm{d} \xi\right) \mathrm{d} s>\frac{1}{\mathrm{e}} .
$$

In 1995, Kusano and Wang [16, Theorem 2] used a variant of the Mahfoud's comparison principle [19] with the ordinary second-order differential equation

$$
\left(\left(x^{\prime}(t)\right)^{\alpha}\right)^{\prime}+\frac{q\left(\tau^{-1}(t)\right)}{\tau^{\prime}\left(\tau^{-1}(t)\right)} x^{\alpha}(t)=0
$$

and proved that $(E)$ is oscillatory if

$$
\liminf _{t \rightarrow \infty} R^{\alpha}\left(\tau(t), t_{0}\right) \int_{t}^{\infty} q(s) \mathrm{d} s>\frac{\alpha^{\alpha}}{(\alpha+1)^{\alpha+1}} .
$$

Condition (1.4) extends the well-known Hille's criterion

$$
\liminf _{t \rightarrow \infty} t \int_{t}^{\infty} q(s) \mathrm{d} s>\frac{1}{4}
$$

for a linear ordinary differential equation

$$
y^{\prime \prime}(t)+q(t) y(t)=0 .
$$

The most oscillation results for $(E)$ existing in the literature use the Riccati transformation to reduce the second-order equation to a first-order Riccati inequality.

In 2006, Sun and Meng [20, Theorem 2.1] improved the oscillation result of Džurina and Stavroulakis [8] by employing the Riccati transformation

$$
\omega(t)=R^{\alpha}\left(\tau(t), t_{0}\right) \frac{r(t)\left(y^{\prime}(t)\right)^{\alpha}}{y^{\alpha}(\tau(t))},
$$


which led to the following criterion for $(E)$ to be oscillatory:

$$
\int_{t_{0}}^{\infty}\left(R^{\alpha}\left(\tau(t), t_{0}\right) q(t)-\left(\frac{\alpha}{\alpha+1}\right)^{\alpha+1} \frac{\tau^{\prime}(t)}{R\left(\tau(t), t_{0}\right) r^{1 / \alpha}(\tau(t))}\right) \mathrm{d} t=\infty .
$$

Similar conditions to (1.7) have been obtained in a number of papers, see, for instance, $[9,10,21,23,24]$ and the references cited therein. It is useful to note (see the proof of Theorem 2.5 below), that we can get Hille-type condition from (1.7).

Despite the fact that the above-mentioned oscillation results were proven by different techniques, they all have in common that their strength depends on sharpness of the estimates for nonoscillatory, say positive solutions of $(E)$.

The purpose of this article is to further study the oscillatory behavior of solutions of $(E)$ and to obtain new criteria which improve the known ones mentioned above. Our approach is essentially based on establishing sharper estimates for positive solutions of $(E)$ than those used in the known works [8-10,14,16,20-24], using an iterative technique, and a comparison principle with first-order delay differential inequalities. If, in some iteration step, the comparison result fails to apply, we are able to improve (in delay case only) conditions of (1.7)-type.

The effectiveness of our results is illustrated by means of various examples.

\section{Main results}

As is customary, we state here that all the functional inequalities considered through the rest of the paper are assumed to hold eventually, that is, they are satisfied for all $t$ large enough.

For a clear and compact presentation of our results, we will adopt the following notation to be used in the whole paper. Let the number $\rho$ be defined by

$$
\rho:=\liminf _{t \rightarrow \infty} \int_{\tau(t)}^{t} q(s) R^{\alpha}\left(\tau(s), t_{0}\right) \mathrm{d} s,
$$

and $\lambda(\eta)$ be the smaller positive root of the transcendental equation

$$
\lambda=e^{\eta \lambda}, \quad 0<\eta \leq 1 / \mathrm{e} .
$$

Also, let us define the sequence of constants $\rho_{k}$ as follows: set

$$
\rho_{1}:=\rho
$$

and, for $\rho_{i} \in(0,1 / \mathrm{e}], i \in \mathbb{N}$, let

$$
\rho_{i+1}:=\liminf _{t \rightarrow \infty} \int_{\tau(t)}^{t} q(s) R_{i}^{\alpha}\left(\tau(s), t_{0}\right) \mathrm{d} s,
$$

where

$$
R_{i}\left(t, t_{0}\right)=R\left(t, t_{0}\right)+\frac{\lambda\left(\rho_{i}\right)}{\alpha} \int_{t_{0}}^{t} R\left(s, t_{0}\right) R^{\alpha}\left(\tau(s), t_{0}\right) q(s) \mathrm{d} s .
$$

We start by stating a simple, but useful result for the first-order delay differential inequality

$$
x^{\prime}(t)+q(t) x(\tau(t)) \leq 0, \quad t \geq t_{0},
$$

where $\tau$ and $q$ are assumed to satisfy (ii) and (iii), respectively.

Lemma 2.1. Let the number $k$ be defined by

$$
k:=\liminf _{t \rightarrow \infty} \int_{\tau(t)}^{t} q(s) \mathrm{d} s .
$$

Suppose that $k>0$ and (2.1) has an eventually positive solution. Then $k \leq 1 / \mathrm{e}$ and

$$
\liminf _{t \rightarrow \infty} \frac{x(\tau(t))}{x(t)} \geq \lambda(k) .
$$


Proof. The proof is almost the same as that given for the corresponding delay differential equation (see [13, Lemma 1]), hence we omit it.

Lemma 2.2. Suppose that $\rho>0$ and $(E)$ has an eventually positive solution. Then $\rho \leq 1 / \mathrm{e}$ and

$$
\liminf _{t \rightarrow \infty} \frac{r(\tau(t))\left(y^{\prime}(\tau(t))\right)^{\alpha}}{r(t)\left(y^{\prime}(t)\right)^{\alpha}} \geq \lambda(\rho) .
$$

Proof. Pick $t_{1} \in\left[t_{0}, \infty\right)$ so that $y(\tau(t))>0$ on $\left[t_{1}, \infty\right)$. Since $y(t)$ is a positive solution of $(E)$, we have

$$
\left(r(t)\left(y^{\prime}(t)\right)^{\alpha}\right)^{\prime}=-q(t) y^{\alpha}(\tau(t)) \leq 0
$$

on $\left[t_{1}, \infty\right)$, which means that $r(t)\left(y^{\prime}(t)\right)^{\alpha}$ is eventually nonincreasing and does not change its sign.

We claim that $r(t)\left(y^{\prime}(t)\right)^{\alpha}>0$ on $\left[t_{1}, \infty\right)$. Indeed, for the sake of contradiction, assume that $r(t)\left(y^{\prime}(t)\right)^{\alpha}<0$ on $\left[t_{1}, \infty\right)$. Then there exists a $t_{1}^{\prime} \geq t_{1}$ such that

$$
r(t)\left(y^{\prime}(t)\right)^{\alpha} \leq r\left(t_{1}^{\prime}\right)\left(y^{\prime}\left(t_{1}^{\prime}\right)\right)^{\alpha}:=c<0 \quad \text { on }\left[t_{1}^{\prime}, \infty\right) .
$$

Integrating the above inequality from $t_{1}^{\prime}$ to $t$ and taking $(i v)$ into account, we have

$$
y(t) \leq y\left(t_{1}^{\prime}\right)+c^{1 / \alpha} \int_{t_{1}^{\prime}}^{t} r^{-1 / \alpha}(s) d s \rightarrow-\infty \quad \text { as } \quad t \rightarrow \infty,
$$

which contradicts the fact that $y(t)$ is a positive solution of $(E)$. Thus, we have

$$
y(t)>0, \quad r(t)\left(y^{\prime}(t)\right)^{\alpha}>0, \quad\left(r(t)\left(y^{\prime}(t)\right)^{\alpha}\right)^{\prime} \leq 0 \quad \text { on }\left[t_{1}, \infty\right) .
$$

Since $r^{1 / \alpha}(t) y^{\prime}(t)$ is nonincreasing, there exists a finite limit

$$
\lim _{t \rightarrow \infty} r^{1 / \alpha}(t) y^{\prime}(t)=\ell \geq 0 .
$$

If we assume $\ell>0$, then $r^{1 / \alpha}(t) y^{\prime}(t) \geq \ell>0$ and $y(t) \geq \ell R\left(t, t_{1}\right)>0$ on $\left[t_{1}, \infty\right)$. Noting that $\rho>0$, we have that

$$
\int_{t_{0}}^{\infty} q(s) R^{\alpha}\left(\tau(s), t_{0}\right) \mathrm{d} s=\infty .
$$

Integrating $(E)$ from $t_{1}$ to $t$ yields

$$
r\left(t_{1}\right)\left(y^{\prime}\left(t_{1}\right)\right)^{\alpha} \geq \ell^{\alpha} \int_{t_{1}}^{t} q(s) R^{\alpha}\left(\tau(s), t_{1}\right) \mathrm{d} s \rightarrow \infty \quad \text { as } \quad t \rightarrow \infty .
$$

This contradiction implies that

$$
\lim _{t \rightarrow \infty} r^{1 / \alpha}(t) y^{\prime}(t)=0 .
$$

On the other hand, it is obvious that

$$
r^{1 / \alpha}(s) y^{\prime}(s) \geq r^{1 / \alpha}(t) y^{\prime}(t) \quad \text { for every } s \in\left[t_{1}, t\right] .
$$

Therefore,

$$
\begin{aligned}
y(t) & =y\left(t_{1}\right)+\int_{t_{1}}^{t} r^{-1 / \alpha}(s) r^{1 / \alpha}(s) y^{\prime}(s) \mathrm{d} s \\
& \geq y\left(t_{1}\right)+r^{1 / \alpha}(t) y^{\prime}(t) R\left(t, t_{1}\right) \\
& =y\left(t_{1}\right)-r^{1 / \alpha}(t) y^{\prime}(t) R\left(t_{1}, t_{0}\right)+r^{1 / \alpha}(t) y^{\prime}(t) R\left(t, t_{0}\right) .
\end{aligned}
$$

Combining (2.3) and (2.4), we have

$$
y(t)>r^{1 / \alpha}(t) y^{\prime}(t) R\left(t, t_{0}\right) \quad \text { on }\left[t_{2}, \infty\right),
$$


for some $t_{2} \in\left[t_{1}, \infty\right)$ large enough. Using (2.5) in $(E)$, it is easy to see that $x(t):=$ $r(t)\left(y^{\prime}(t)\right)^{\alpha}$ is a positive solution of the first-order delay differential inequality

$$
x^{\prime}(t)+q(t) R^{\alpha}\left(\tau(t), t_{0}\right) x(\tau(t))<0 .
$$

To complete the proof, it suffices to apply Lemma 2.1 to (2.6).

Application of Lemma 2.2 allows us to obtain various important oscillation results. Theorem 2.3 below is a simple generalization of (1.2) for a half-linear differential equation, while Theorems 2.4 and 2.5 essentially improve the known criteria (1.7) and (1.4), respectively.

Theorem 2.3. If $\rho>1 / \mathrm{e}$, then $(E)$ is oscillatory.

Theorem 2.4. Assume that $0<\rho \leq 1 /$ e. If

$$
\begin{aligned}
\limsup _{t \rightarrow \infty} & \int_{t_{0}}^{t}\left(R^{\alpha}\left(\tau(s), t_{0}\right) q(s)\right. \\
& \left.-\left(\frac{\alpha}{\alpha+1}\right)^{\alpha+1} \frac{1}{(\lambda(\rho)-\epsilon)} \frac{\tau^{\prime}(s)}{R\left(\tau(s), t_{0}\right) r^{1 / \alpha}(\tau(s))}\right) \mathrm{d} s=\infty
\end{aligned}
$$

for some $\epsilon>0$, then $(E)$ is oscillatory.

Proof. Suppose to the contrary that $(E)$ has a nonoscillatory solution $y(t)$ on $\left[t_{0}, \infty\right)$. Without loss of generality, we can assume that there exists a $t_{1} \geq t_{0}$ such that $y(t)>0$ and $y(\tau(t))>0$ on $\left[t_{1}, \infty\right)$. Define $\omega(t)$ as in (1.6), i.e.,

$$
\omega(t)=R^{\alpha}\left(\tau(t), t_{0}\right) \frac{r(t)\left(y^{\prime}(t)\right)^{\alpha}}{y^{\alpha}(\tau(t))} .
$$

We see that $\omega>0$ for $t \geq t_{1}$. Differentiating (2.8) and using $(E)$, we get

$$
\begin{aligned}
\omega^{\prime}(t)= & \frac{\alpha \tau^{\prime}(t) R^{\alpha-1}\left(\tau(t), t_{0}\right)}{r^{1 / \alpha}(\tau(t))} \frac{r(t)\left(y^{\prime}(t)\right)^{\alpha}}{y^{\alpha}(\tau(t))}-R^{\alpha}\left(\tau(t), t_{0}\right) \frac{\left(r(t)\left(y^{\prime}(t)\right)^{\alpha}\right)^{\prime}}{y^{\alpha}(\tau(t))} \\
& -R^{\alpha}\left(\tau(t), t_{0}\right) \frac{\alpha r(t)\left(y^{\prime}(t)\right)^{\alpha} y^{\prime}(\tau(t)) \tau^{\prime}(t)}{y^{\alpha+1}(\tau(t))} \\
= & \frac{\alpha \tau^{\prime}(t)}{R\left(\tau(t), t_{0}\right) r^{1 / \alpha}(\tau(t))} \omega(t)-R^{\alpha}\left(\tau(t), t_{0}\right) q(t) \\
& -R^{\alpha}\left(\tau(t), t_{0}\right) \frac{\alpha r(t)\left(y^{\prime}(t)\right)^{\alpha} y^{\prime}(\tau(t)) \tau^{\prime}(t)}{y^{\alpha+1}(\tau(t))} .
\end{aligned}
$$

Lemma 2.2 implies that, for each $\epsilon>0$, there is $t_{2} \in\left[t_{1}, \infty\right)$ large enough such that

$$
y^{\prime}(\tau(t)) \geq\left((\lambda(\rho)-\epsilon) \frac{r(t)}{r(\tau(t))}\right)^{1 / \alpha} y^{\prime}(t), \quad \text { on }\left[t_{2}, \infty\right) .
$$

Combining (2.8)-(2.10), we obtain

$$
\begin{aligned}
\omega^{\prime}(t) \leq & \frac{\alpha \tau^{\prime}(t)}{R\left(\tau(t), t_{0}\right) r^{1 / \alpha}(\tau(t))} \omega(t)-R^{\alpha}\left(\tau(t), t_{0}\right) q(t) \\
& -\alpha \tau^{\prime}(t)\left(\frac{\lambda(\rho)-\epsilon}{R^{\alpha}\left(\tau(t), t_{0}\right) r(\tau(t))}\right)^{1 / \alpha} \omega^{(\alpha+1) / \alpha}(t) .
\end{aligned}
$$

Using the inequality

$$
A u-B u^{(\alpha+1) / \alpha} \leq \frac{\alpha^{\alpha}}{(\alpha+1)^{\alpha+1}} \frac{A^{\alpha+1}}{B^{\alpha}}, \quad A \geq 0, B>0, u \geq 0
$$


with

in (2.11), we obtain

$$
A:=\frac{\alpha \tau^{\prime}(t)}{R\left(\tau(t), t_{0}\right) r^{1 / \alpha}(\tau(t))} \quad \text { and } \quad B:=\alpha \tau^{\prime}(t)\left(\frac{\lambda(\rho)-\epsilon}{R^{\alpha}\left(\tau(t), t_{0}\right) r(\tau(t))}\right)^{1 / \alpha}
$$

$$
w^{\prime}(t) \leq-R^{\alpha}\left(\tau(t), t_{0}\right) q(t)+\left(\frac{\alpha}{\alpha+1}\right)^{\alpha+1} \frac{1}{\lambda(\rho)-\epsilon} \frac{\tau^{\prime}(t)}{R\left(\tau(t), t_{0}\right) r^{1 / \alpha}(\tau(t))} .
$$

Integrating the above inequality from $t_{2}$ to $t$, we arrive at

$$
\begin{aligned}
& \int_{t_{2}}^{t}\left(R^{\alpha}\left(\tau(s), t_{0}\right) q(s)-\left(\frac{\alpha}{\alpha+1}\right)^{\alpha+1} \frac{1}{\lambda(\rho)-\epsilon} \frac{\tau^{\prime}(s)}{R\left(\tau(s), t_{0}\right) r^{1 / \alpha}(\tau(s))}\right) \mathrm{d} s \\
& \leq \omega\left(t_{2}\right)-\omega(t) \leq \omega\left(t_{2}\right),
\end{aligned}
$$

which contradicts (2.7) as $t \rightarrow \infty$. The proof is complete.

Theorem 2.5. Assume that $0<\rho \leq 1 /$ e. If

$$
\liminf _{t \rightarrow \infty} R^{\alpha}\left(\tau(t), t_{0}\right) \int_{t}^{\infty} q(s) \mathrm{d} s>\frac{1}{\lambda(\rho)} \frac{\alpha^{\alpha}}{(\alpha+1)^{\alpha+1}},
$$

then $(E)$ is oscillatory.

Proof. It suffices to prove that (2.12) implies (2.7). If we admit that (2.7) fails, then for all $\tilde{\varepsilon}>0$ there exists a $t_{1} \in\left[t_{0}, \infty\right)$ such that for any $t \geq t_{1}$,

$$
\int_{t}^{\infty}\left(R^{\alpha}\left(\tau(s), t_{0}\right) q(s)-\frac{1}{\lambda(\rho)-\epsilon}\left(\frac{\alpha}{\alpha+1}\right)^{\alpha+1} \frac{\tau^{\prime}(s)}{R\left(\tau(s), t_{0}\right) r^{1 / \alpha}(\tau(s))}\right) \mathrm{d} s<\tilde{\epsilon} .
$$

Since $R\left(\tau(t), t_{0}\right)$ is increasing, it is easy to see that

$$
\begin{aligned}
& R^{\alpha}\left(\tau(t), t_{0}\right) \times \\
& \times \int_{t}^{\infty}\left(q(s)-\frac{1}{\lambda(\rho)-\epsilon}\left(\frac{\alpha}{\alpha+1}\right)^{\alpha+1} \frac{\tau^{\prime}(s)}{R^{\alpha+1}\left(\tau(s), t_{0}\right) r^{1 / \alpha}(\tau(s))}\right) \mathrm{d} s<\tilde{\epsilon},
\end{aligned}
$$

or

Hence,

$$
R^{\alpha}\left(\tau(t), t_{0}\right) \int_{t}^{\infty}\left(q(s)+\frac{1}{\lambda(\rho)-\epsilon} \frac{\alpha^{\alpha}}{(\alpha+1)^{\alpha+1}}\left(\frac{1}{R^{\alpha}\left(\tau(s), t_{0}\right)}\right)^{\prime}\right) \mathrm{d} s<\tilde{\epsilon}
$$

$$
R^{\alpha}\left(\tau(t), t_{0}\right) \int_{t}^{\infty} q(s) \mathrm{d} s<\tilde{\epsilon}+\frac{1}{\lambda(\rho)-\epsilon} \frac{\alpha^{\alpha}}{(\alpha+1)^{\alpha+1}}
$$

for all $\tilde{\epsilon}>0$, which contradicts (2.12). The proof is complete.

Lemma 2.6. Suppose that $\rho>0$ and $(E)$ has an eventually positive solution. Then, for any $k \in \mathbb{N}, 0<\rho_{k} \leq 1 / \mathrm{e}$ and

$$
\liminf _{t \rightarrow \infty} \frac{r(\tau(t))\left(y^{\prime}(\tau(t))\right)^{\alpha}}{r(t)\left(y^{\prime}(t)\right)^{\alpha}} \geq \lambda\left(\rho_{k}\right) .
$$

Proof. By Lemma 2.2, it is clear that the statement holds for $k=1$, i.e., $\rho_{1} \leq 1$ /e and, for each $\epsilon>0$, there is $t_{2} \in\left[t_{1}, \infty\right)$ such that

$$
\frac{r(\tau(t))\left(y^{\prime}(\tau(t))\right)^{\alpha}}{r(t)\left(y^{\prime}(t)\right)^{\alpha}} \geq \lambda\left(\rho_{1}\right)-\epsilon, \quad \text { on }\left[t_{2}, \infty\right) .
$$

Now, employing the chain rule

$$
\left(r(t)\left(y^{\prime}(t)\right)^{\alpha}\right)^{\prime}=\alpha\left(r^{1 / \alpha} y^{\prime}(t)\right)^{\alpha-1}\left(r^{1 / \alpha} y^{\prime}(t)\right)^{\prime}
$$

in the equality

$$
\left(y(t)-r^{1 / \alpha}(t) y^{\prime}(t) R\left(t, t_{0}\right)\right)^{\prime}=-R\left(t, t_{0}\right)\left(r^{1 / \alpha} y^{\prime}(t)\right)^{\prime}
$$


we get

$$
\left(y(t)-r^{1 / \alpha}(t) y^{\prime}(t) R\left(t, t_{0}\right)\right)^{\prime}=-\frac{1}{\alpha} R\left(t, t_{0}\right)\left(r^{1 / \alpha} y^{\prime}(t)\right)^{1-\alpha}\left(r(t)\left(y^{\prime}(t)\right)^{\alpha}\right)^{\prime},
$$

which, by virtue of $(E)$, becomes

$$
\left(y(t)-r^{1 / \alpha}(t) y^{\prime}(t) R\left(t, t_{0}\right)\right)^{\prime}=\frac{1}{\alpha} R\left(t, t_{0}\right)\left(r^{1 / \alpha} y^{\prime}(t)\right)^{1-\alpha} q(t) y^{\alpha}(\tau(t)) .
$$

Integrating (2.15) from $t_{2}$ to $t$ yields to

$$
\phi(t)=\phi\left(t_{2}\right)+\frac{1}{\alpha} \int_{t_{2}}^{t} R\left(s, t_{0}\right)\left(r^{1 / \alpha} y^{\prime}(s)\right)^{1-\alpha} q(s) y^{\alpha}(\tau(s)) \mathrm{d} s,
$$

where we set $\phi(t)=y(t)-r^{1 / \alpha}(t) y^{\prime}(t) R\left(t, t_{0}\right)$. It is clear from $(2.5)$ that $\phi(t)$ is positive on $\left[t_{2}, \infty\right)$. Now, using $(2.5)$ and (2.14) in (2.16), we arrive at

$$
\begin{aligned}
\phi(t) \geq \phi\left(t_{2}\right)+ & \frac{1}{\alpha} \int_{t_{2}}^{t} R\left(s, t_{0}\right)\left(r^{1 / \alpha}(s) y^{\prime}(s)\right)^{1-\alpha} \times \\
& \times q(s) r(\tau(s))\left(y^{\prime}(\tau(s))\right)^{\alpha} R^{\alpha}\left(\tau(s), t_{0}\right) \mathrm{d} s \\
\geq \phi\left(t_{2}\right)+ & \frac{\lambda\left(\rho_{1}\right)-\epsilon}{\alpha} \int_{t_{2}}^{t} R\left(s, t_{0}\right)\left(r^{1 / \alpha}(s) y^{\prime}(s)\right)^{1-\alpha} \times \\
& \times q(s) r(s)\left(y^{\prime}(s)\right)^{\alpha} R^{\alpha}\left(\tau(s), t_{0}\right) \mathrm{d} s \\
=\phi\left(t_{2}\right)+ & \frac{\lambda\left(\rho_{1}\right)-\epsilon}{\alpha} \int_{t_{2}}^{t} R\left(s, t_{0}\right) R^{\alpha}\left(\tau(s), t_{0}\right) r^{1 / \alpha}(s) y^{\prime}(s) q(s) \mathrm{d} s .
\end{aligned}
$$

Using the nondecreasing character of $r(t)\left(y^{\prime}(t)\right)^{\alpha}$ in the latter inequality, we obtain

$$
\begin{aligned}
\phi(t) \geq & \phi\left(t_{2}\right)+\frac{\lambda\left(\rho_{1}\right)-\epsilon}{\alpha} r^{1 / \alpha}(t) y^{\prime}(t) \int_{t_{2}}^{t} R\left(s, t_{0}\right) R^{\alpha}\left(\tau(s), t_{0}\right) q(s) \mathrm{d} s \\
= & \phi\left(t_{2}\right)-\frac{\lambda\left(\rho_{1}\right)-\epsilon}{\alpha} r^{1 / \alpha}(t) y^{\prime}(t) \int_{t_{0}}^{t_{2}} R\left(s, t_{0}\right) R^{\alpha}\left(\tau(s), t_{0}\right) q(s) \mathrm{d} s \\
& +\frac{\lambda\left(\rho_{1}\right)-\epsilon}{\alpha} r^{1 / \alpha}(t) y^{\prime}(t) \int_{t_{0}}^{t} R\left(s, t_{0}\right) R^{\alpha}\left(\tau(s), t_{0}\right) q(s) \mathrm{d} s .
\end{aligned}
$$

By virtue of (2.3) and the positivity of $\phi$, we have

$$
\phi(t)>\frac{\lambda\left(\rho_{1}\right)-\epsilon}{\alpha} r^{1 / \alpha}(t) y^{\prime}(t) \int_{t_{0}}^{t} R\left(s, t_{0}\right) R^{\alpha}\left(\tau(s), t_{0}\right) q(s) \mathrm{d} s \quad \text { on }\left[t_{3}, \infty\right),
$$

for some $t_{3} \in\left[t_{2}, \infty\right)$ large enough. Hence,

$$
y(t)>r^{1 / \alpha}(t) y^{\prime}(t)\left(R\left(t, t_{0}\right)+\frac{\lambda\left(\rho_{1}\right)-\epsilon}{\alpha} \int_{t_{0}}^{t} R\left(s, t_{0}\right) R^{\alpha}\left(\tau(s), t_{0}\right) q(s) \mathrm{d} s\right)
$$

or

where

$$
y(t)>r^{1 / \alpha}(t) y^{\prime}(t) R_{1}\left(t, t_{0}, \epsilon\right) \quad \text { on }\left[t_{3}, \infty\right),
$$

$$
R_{1}\left(t, t_{0}, \epsilon\right)=R\left(t, t_{0}\right)+\frac{\lambda\left(\rho_{1}\right)-\epsilon}{\alpha} \int_{t_{0}}^{t} R\left(s, t_{0}\right) R^{\alpha}\left(\tau(s), t_{0}\right) q(s) \mathrm{d} s .
$$

Using (2.20) in $(E)$, we see that $x(t):=r(t)\left(y^{\prime}(t)\right)^{\alpha}$ is a positive solution of the first-order delay differential inequality

$$
x^{\prime}(t)+R_{1}^{\alpha}\left(\tau(t), t_{0}, \epsilon\right) q(t) x(\tau(t))<0 .
$$

Applying Lemma 2.1 to (2.21), it is clear that the conclusion holds for $k=2$, that is, $\rho_{2} \leq 1 /$ e and, for each $\epsilon>0$, there is $t_{4} \in\left[t_{1}, \infty\right)$ such that

$$
\frac{r(\tau(t))\left(y^{\prime}(\tau(t))\right)^{\alpha}}{r(t)\left(y^{\prime}(t)\right)^{\alpha}} \geq \lambda\left(\rho_{2}\right)-\epsilon, \quad \text { on }\left[t_{4}, \infty\right) .
$$


Repeating the above process with (2.5) being replaced by (2.20), one can show that

$$
y(t)>r^{1 / \alpha}(t) y^{\prime}(t) R_{2}\left(t, t_{0}, \epsilon\right) \text { on }\left[t_{5}, \infty\right),
$$

for some $t_{5} \in\left[t_{4}, \infty\right)$, where

$$
R_{2}\left(t, t_{0}, \epsilon\right)=R\left(t, t_{0}\right)+\frac{\lambda\left(\rho_{2}\right)-\epsilon}{\alpha} \int_{t_{0}}^{t} R\left(s, t_{0}\right) R^{\alpha}\left(\tau(s), t_{0}\right) q(s) \mathrm{d} s .
$$

Using (2.20) in $(E)$ and applying Lemma 2.1 to a resulting inequality, we see that the Lemma conclusion holds for $k=3$. By induction, it is not hard to show that the same conclusion holds for any $k \in \mathbb{N}$. The proof is complete.

Using Lemma 2.6 instead of Lemma 2.2, we are ready to improve Theorems 2.3-2.5. Since the proofs are the same, we omit them.

Theorem 2.7. If $\rho_{k}>1 /$ e for some $k \in \mathbb{N}$, then $(E)$ is oscillatory.

Theorem 2.8. Assume that $0<\rho_{i} \leq 1 / \mathrm{e}, i=1,2, \ldots, k$, for some $k \in \mathbb{N}$. If

$$
\begin{aligned}
\limsup _{t \rightarrow \infty} & \int_{t_{0}}^{t}\left(R^{\alpha}\left(\tau(s), t_{0}\right) q(s)\right. \\
& \left.-\left(\frac{\alpha}{\alpha+1}\right)^{\alpha+1} \frac{1}{\left(\lambda\left(\rho_{k}\right)-\epsilon\right)} \frac{\tau^{\prime}(s)}{R\left(\tau(s), t_{0}\right) r^{1 / \alpha}(\tau(s))}\right) \mathrm{d} s=\infty
\end{aligned}
$$

for some $\epsilon>0$, then $(E)$ is oscillatory.

Theorem 2.9. Assume that $0<\rho_{i} \leq 1 / \mathrm{e}, i=1,2, \ldots, k$, for some $k \in \mathbb{N}$. If

$$
\liminf _{t \rightarrow \infty} R^{\alpha}\left(\tau(t), t_{0}\right) \int_{t}^{\infty} q(s) \mathrm{d} s>\frac{1}{\lambda\left(\rho_{k}\right)} \frac{\alpha^{\alpha}}{(\alpha+1)^{\alpha+1}},
$$

then $(E)$ is oscillatory.

Finally, we give an example to illustrate the efficiency of our results.

Example 2.10. Consider the second-order delay differential equation of the Euler type:

$$
\left(\left(y^{\prime}(t)\right)^{\alpha}\right)^{\prime}+\frac{q_{0}}{t^{\alpha+1}} y^{\alpha}(m t)=0, \quad t \geq 1,
$$

where $\alpha$ is a quotient of odd positives integers, $q_{0}>0, m \in(0,1)$.

Note that the known condition (1.7) (or (1.4)) requires

$$
q_{0} m^{\alpha}>\frac{\alpha^{\alpha}}{(\alpha+1)^{\alpha+1}}
$$

for (2.26) to be oscillatory.

By Theorem 2.3, we have that Eq. (2.26) is oscillatory if

$$
\rho:=q_{0} m^{\alpha} \ln \frac{1}{m}>\frac{1}{\mathrm{e}} .
$$

Now consider the case that (2.28) fails, that is, if $\rho \leq 1 /$ e. By Theorem 2.4 (or Theorem $2.5)$, we deduce that (2.26) is oscillatory if

$$
q_{0} m^{\alpha}>\frac{1}{\lambda(\rho)} \frac{\alpha^{\alpha}}{(\alpha+1)^{\alpha+1}} .
$$

Since $\lambda(\rho) \in[1, \mathrm{e})$, our result improves $(2.27)$.

Next, let us illustrate how Theorems 2.7 and 2.8 apply when both condition (2.28) and (2.29) fail. By Theorem 2.7, equation (2.26) is oscillatory if, for some $k \in \mathbb{N}$,

$$
\rho_{k}>\frac{1}{\mathrm{e}},
$$

where

$$
\rho_{1}:=\rho
$$


and, for $\rho_{i} \in(0,1 / \mathrm{e}], i \in \mathbb{N}$,

$$
\rho_{i+1}:=q_{0} m^{\alpha}\left(1+\frac{\lambda\left(\rho_{i}\right)}{\alpha} m^{\alpha} q_{0}\right)^{\alpha} \ln \frac{1}{m}, \quad i \in \mathbb{N} .
$$

If, in $k$ th iteration step, condition (2.30) fails, then, by Theorem 2.8 (or Theorem 2.9), we have that (2.26) is oscillatory if

$$
q_{0} m^{\alpha}>\frac{1}{\lambda\left(\rho_{k}\right)} \frac{\alpha^{\alpha}}{(\alpha+1)^{\alpha+1}} .
$$

Now, let us consider a particular case of (2.26), namely,

$$
\left(\left(y^{\prime}(t)\right)^{3}\right)^{\prime}+\frac{11}{t^{4}} y^{3}(0.2 t)=0 .
$$

Note that condition (2.27) is not applicable, since $0.088 \ngtr 0.105469$. Using the definition of $\rho_{k}$, we get $\rho_{1}=0.141631 \ngtr 1 / \mathrm{e}, \rho_{2}=0.156883 \ngtr 1 / \mathrm{e}$. Then condition $(2.31)$ with $k=2$ gives $0.106376>0.105469$. Hence, by Theorem $2.8,(2.32)$ is oscillatory.

Finally, we consider another particular case of (2.26), namely,

$$
\left(\left(y^{\prime}(t)\right)^{1 / 3}\right)^{\prime}+\frac{0.4}{t^{4 / 3}} y^{1 / 3}(0.4 t)=0 .
$$

Note that condition (2.27) is not applicable, since $0.294723 \ngtr 0.47247$. Using the definition of $\rho_{k}$, we get $\rho_{1}=0.270052 \ngtr 1 / \mathrm{e}, \rho_{2}=0.357776 \ngtr 1 / \mathrm{e}, \rho_{3}=0.386561>1 / \mathrm{e}$. Thus, condition (2.30) is satisfied for $k=3$, and by Theorem 2.7 , we conclude that (2.33) is oscillatory.

We remark that none of the oscillation criteria presented in $[8-10,14,16,20-24]$ can be applied to equation (2.32) or (2.33).

Remark 2.11. The results presented in this paper strongly depend on the properties of first-order delay differential equations. An interesting problem for further research is to establish different iterative techniques for testing oscillations in $(E)$ independently on the constant $1 / \mathrm{e}$.

Acknowledgment. The authors would like to thank the editors and three referees for the constructive remarks which improved the presentation of the paper. The work of the second author was supported by the grant project KEGA 035TUKE-4/2017.

\section{References}

[1] R. P. Agarwal, M. Bohner and W.-T. Li, Nonoscillation and oscillation: theory for functional differential equations, Monographs and Textbooks in Pure and Applied Mathematics 267, Marcel Dekker, Inc., New York, 2004.

[2] R. P. Agarwal, S. R. Grace and D. O'Regan, Oscillation theory for second order linear, half-linear, superlinear and sublinear dynamic equations, Kluwer Academic Publishers, Dordrecht, 2002.

[3] R. P. Agarwal, S. R. Grace and D. O'Regan, Oscillation theory for second order dynamic equations, Series in Mathematical Analysis and Applications 5, Taylor \& Francis, Ltd., London, 2003.

[4] R. P. Agarwal, S. R. Grace and D. O'Regan, Oscillation theory for difference and functional differential equations, Springer Science \& Business Media, 2013.

[5] R. P. Agarwal, C. Zhang and T. Li, Some remarks on oscillation of second order neutral differential equations, Appl. Math. Comput. 274, 178-181, 2016.

[6] O. Došlý and P. Rehák, Half-linear differential equations, North-Holland Mathematics Studies 202, Elsevier Science B.V., Amsterdam, 2005.

[7] J. Džurina and I. Jadlovská, A note on oscillation of second-order delay differential equations, Appl. Math. Lett. 69, 126-132, 2017. 
[8] J. Džurina and I. P. Stavroulakis, Oscillation criteria for second-order delay differential equations, Appl. Math. Comput. 140 (2-3), 445-453, 2003.

[9] L. Erbe, A. Peterson and S. H. Saker, Kamenev-type oscillation criteria for secondorder linear delay dynamic equations, Dynam. Systems Appl. 15 (1), 65-78, 2006.

[10] S. Fišnarová and R. Mařík, Oscillation of half-linear differential equations with delay, Abstr. Appl. Anal. 2013, Article ID: 583147, 1-6, 2013.

[11] I. Győri and G. Ladas, Oscillation theory of delay differential equations, Oxford Mathematical Monographs, The Clarendon Press, Oxford University Press, New York, 1991.

[12] Z. Han, T. Li, S. Sun and Y. Sun, Remarks on the paper [Appl. Math. Comput. 207 (2009) 388-396] [mr2489110], Appl. Math. Comput. 215 (11), 3998-4007, 2010.

[13] J. Jaroš and I. P. Stavroulakis, Oscillation tests for delay equations, Rocky Mountain J. Math. 29 (1), 197-207, 1999.

[14] R. Koplatadze, G. Kvinikadze and I. P. Stavroulakis, Oscillation of second order linear delay differential equations, Funct. Differ. Equ. 7 (1-2), 121-145, 2000.

[15] R. G. Koplatadze, Criteria for the oscillation of solutions of differential inequalities and second-order equations with retarded argument, Tbiliss. Gos. Univ. Inst. Prikl. Mat. Trudy, 17, 104-121, 1986.

[16] T. Kusano and J. Wang, Oscillation properties of half-linear functional-differential equations of the second order, Hiroshima Math. J. 25 (2), 371-385, 1995.

[17] T. Li, Y. V. Rogovchenko and C. Zhang, Oscillation of second-order neutral differential equations, Funkcial. Ekvac. 56 (1), 111-120, 2013.

[18] T. Li, Y. V. Rogovchenko and C. Zhang, Oscillation results for second-order nonlinear neutral differential equations, Adv. Difference Equ. 2013 (336), 1-13, 2013.

[19] W. E. Mahfoud, Comparison theorems for delay differential equations, Pacific J. Math. 83 (1), 187-197, 1979.

[20] Y. G. Sun and F. W. Meng, Note on the paper of Džurina and Stavroulakis: "Oscillation criteria for second-order delay differential equations" [Appl. Math. Comput. 140 (2003), no. 2-3, 445-453; mr1953915], Appl. Math. Comput. 174 (2), 1634-1641, 2006.

[21] A. Tiryaki, Oscillation criteria for a certain second-order nonlinear differential equations with deviating arguments, Electron. J. Qual. Theory Differ. Equ. 2009 (61), $1-11,2009$.

[22] J. J. Wei, Oscillation of second order delay differential equation, Ann. Differential Equations, 4 (4), 473-478, 1988.

[23] H. Wu, L. Erbe and A. Peterson, Oscillation of solution to second-order half-linear delay dynamic equations on time scales, Electron. J. Differential Equations, 2016 (71), $1-15,2016$.

[24] R. Xu and F. Meng, Some new oscillation criteria for second order quasi-linear neutral delay differential equations, Appl. Math. Comput. 182 (1), 797-803, 2006.

[25] L. Ye and Z. Xu, Oscillation criteria for second order quasilinear neutral delay differential equations, Appl. Math. Comput. 207 (2), 388-396, 2009.

[26] C. Zhang, R. P. Agarwal, M. Bohner and T. Li, Oscillation of second-order nonlinear neutral dynamic equations with noncanonical operators, Bull. Malays. Math. Sci. Soc. 38 (2), 761-778, 2015. 